\title{
Développement durable et agriculture durable : sens du concept de « durabilité » à travers la presse régionale et le discours des agriculteurs
}

Sustainable development and sustainable agriculture : the definition of "sustainability" in a daily local newspaper and famers' discourse

Lise Bihannic et Élisabeth Michel-Guillou

\section{OpenEdition}

\section{Journals}

Édition électronique

URL : http://journals.openedition.org/developpementdurable/9076

DOI : 10.4000/developpementdurable.9076

ISSN : 1772-9971

Éditeur

Association DD\&T

Référence électronique

Lise Bihannic et Élisabeth Michel-Guillou, « Développement durable et agriculture durable : sens du concept de "durabilité » à travers la presse régionale et le discours des agriculteurs », Développement durable et territoires [En ligne], Vol. 2, nº 3 | Décembre 2011, mis en ligne le 03 décembre 2011, consulté le 30 avril 2019. URL : http://journals.openedition.org/developpementdurable/9076 ; DOI : 10.4000/ developpementdurable.9076

Ce document a été généré automatiquement le 30 avril 2019.

Développement Durable et Territoires est mis à disposition selon les termes de la licence Creative Commons Attribution - Pas d'Utilisation Commerciale 4.0 International. 


\title{
Développement durable et agriculture durable : sens du concept de " durabilité » à travers la presse régionale et le discours des agriculteurs
}

\author{
Sustainable development and sustainable agriculture : the definition of \\ "sustainability" in a daily local newspaper and famers' discourse
}

Lise Bihannic et Élisabeth Michel-Guillou

1 Le concept de développement durable est, de nos jours, omniprésent dans les discours. En lien avec le rapport Brundtland, il est communément définit comme «un mode de développement qui répond aux besoins du présent sans compromettre la capacité des générations futures de répondre aux leurs. " (CMED, 2005, p. 51). Il est formalisé à partir de trois piliers. Le pilier écologique ou environnemental a trait au respect de l'environnement, à la dynamique naturelle, à la gestion des ressources naturelles. Le pilier économique renvoie à l'efficacité, la dynamique, la cohérence économique. Le pilier social porte sur les questions d'équité sociale, de solidarité, de lien social, d'identité culturelle. Viser une orientation en matière de développement durable permettrait alors de concilier croissance et efficacité économique, équité et progrès social, tout en préservant l'environnement. Malgré une certaine valorisation sociale (Pol, 2003), le concept possède ses détracteurs et fait l'objet de nombreuses critiques, la principale reposant sur l'incompatibilité des termes «développement » et "durable ». Les acteurs, dénonçant cet oxymore, suggèrent que la préservation de l'environnement ne peut être conciliable avec la croissance économique actuelle. Il est donc objet d'enjeux, de débats et de prises de position parfois divergentes selon les groupes. Ces divergences peuvent, par exemple, conduire certaines personnes ou groupes à privilégier une dimension du développement durable au détriment des autres. 
2 De par ses modes de production intensifs, sa fonction de production alimentaire et l'utilisation particulière qu'elle fait des ressources environnementales, l'agriculture est l'un des domaines les plus concernés par la question du développement durable (Charvet, 2007 ; Humbert, 2008). En effet, le développement agricole des 50 dernières années a engendré des dysfonctionnements au niveau social (baisse de la main-d'œuvre, etc.), économique (accroissement des inégalités, revenu subventionné, etc.) et écologique (érosion des sols, pollution de l'eau, etc.) (Jollivet, 2001). La présente recherche porte sur la construction du sens des concepts de développement durable et d'agriculture durable, à la fois du point de vue du sens commun à travers sa diffusion dans la presse et également pour les agriculteurs, acteurs en prise avec ces questions de durabilité. Nous prendrons l'exemple de plusieurs agricultures (conventionnelle, biologique, de conservation des sols) et analyserons le contenu des corpus, selon une approche psychosociologique et environnementale du rapport de l'individu à l'environnement.

\section{Le développement durable : une forme de construction sociale}

3 S'intéressant à la complexité des rapports homme-environnement, de nombreux auteurs en psychologie environnementale se sont intéressés à la manière dont cette discipline, en tant que science du comportement humain, pouvait contribuer à la durabilité des écosystèmes (Bonnes, Bonaiuto, 2002 ; Moser, 2009 ; Oskamp, 2000 ; Pol, 2003 ; Schmuck, Schultz, 2002; Weiss, Girandola, 2010). Accordant une place prépondérante à l'Homme dans la dégradation des ressources, la psychologie environnementale se propose de comprendre les facteurs qui freinent ou favorisent l'engagement des individus dans des actions pro-environnementales. Parce que les comportements écologiques n'obéissent pas à une logique déterministe simple (Moser, 2009; Ratiu, 2003) et que les ressources naturelles font partie de biens communs, une approche individuelle du rapport à l'environnement trouve rapidement ses limites (Michel-Guillou, Weiss, 2007). La dimension collective et socioculturelle du rapport à l'environnement s'avère essentielle dans la compréhension de l'adoption, ou non, de comportements pro-environnementaux (Bonnes, Bonaiuto, 2002).

4 La théorie des représentations sociales permet l'analyse de cette médiation et des interrelations complexes entre l'homme et son environnement physique et social. Jodelet (1999) définit les représentations sociales comme «une forme de connaissance, socialement élaborée et partagée, ayant une visée pratique et concourant à la construction d'une réalité commune à un ensemble social. Également désignée comme "savoir de sens commun" ou encore "savoir naif ", "naturel», cette forme de connaissance est distinguée, entre autres, de la connaissance scientifique » (p. 53). Situées à l'interface entre le psychologique et le social, les représentations sociales sont à la base de notre système de pensée. Moscovici (1976) a montré comment différents groupes sociaux élaborent une représentation sociale d'un objet encore peu connu, de manière à ce qu'elle soit en concordance avec leur système de pensée préexistant (attentes, valeurs, croyances, etc.). L'auteur insiste également sur l'importance du rôle des modes de communication et notamment de la presse dans l'élaboration des représentations sociales. Se basant sur ces travaux, Doise (1985) propose de considérer les représentations sociales comme des prises de position symboliques qui organisent les rapports sociaux et qui diffèrent selon leur imbrication. 
5 En définitive, chaque personne, de par ces appartenances de groupe, se construit une représentation de la réalité qui lui permet de comprendre, d'interpréter, de maitriser, de rendre signifiant le monde qui l'entoure. En rapport à un même objet, il existe donc potentiellement autant de réalités que de groupes ou de communautés qui y sont confrontés. Concernant le développement durable, les préoccupations des uns ne sont pas toujours celles des autres, c'est pourquoi chaque acteur ou groupe, en prise avec des questions liées au développement durable, peut avoir sa propre représentation du concept.

\section{Objectifs de l'étude}

6 Le concept de développement durable est aujourd'hui largement répandu dans la société et son appropriation par la classe politique ainsi que sa médiatisation lui ont permis d'être socialement valorisé (Pol, 2003). Dans ce contexte, l'agriculture occupe une place importante car elle joue un rôle primordial dans la gestion de nos espaces ruraux (MichelGuillou \& Moser, 2006; Moser, 2009). Mais du fait de ce rapport privilégié à la nature, les agriculteurs sont souvent tenus pour responsables de certains faits de pollution et des pressions (sociales, économiques, etc.) s'exercent sur eux pour les contraindre à une modification des pratiques. L'agriculture intensive est particulièrement condamnée et remise en cause. Depuis les années 1990, des alternatives à ce système de production se mettent en place, notamment à l'initiative de certains groupes d'agriculteurs conscients que le système ne peut perdurer. L'enjeu pour une majorité de ces groupes est de conserver une agriculture compétitive économiquement, tout en garantissant une production respectueuse de l'environnement et en réconciliant l'agriculture et la société. L'agriculture est donc particulièrement concernée par les questions liées à la «durabiltié » (Charvet, 2007; Humbert, 2008) que ces questions soient en lien avec des enjeux économiques (maintenir une exploitation, un revenu, etc.), environnementaux (maintenir/préserver des ressources naturelles, etc.) et/ou sociaux (conserver un emploi, un niveau de vie, permettre une vie sociale, etc.).

7 En lien avec la théorie précédemment développée, ce travail s'intéresse d'une part au sens que les concepts de développement durable et d'agriculture durable recouvrent pour les agriculteurs et d'autre part, à leur diffusion au sein de la presse régionale, généraliste et spécialisée. Il s'agit donc d'étudier la représentation sociale de ces concepts qui agissent comme un guide pour l'action. Dans la mesure où, par définition, les représentations sociales s'élaborent et se transmettent par les canaux de communication, nous mettrons en parallèle le contenu des articles consacrés à ces thèmes avec le discours recueilli auprès des interviewés. En outre, la prise de conscience des problèmes environnementaux et la multiplication des démarches en faveur de l'environnement implique de considérer la diversité des agricultures et des pratiques au sein du monde agricole (Charvet, 2007). Nous pouvons donc supposer que chaque groupe, en fonction de ses pratiques intimement liées avec ses représentations, s'appropriera différemment ces concepts. 


\section{Méthode}

\subsection{La procédure d'enquête et l'échantillon}

L'étude présentée a été menée en Bretagne au début de l'année 2008. Pour comprendre de manière approfondie le sens que les agriculteurs donnent au développement durable et à l'agriculture durable, des entretiens semi-directifs ont été menés. Ces entretiens se structurent autour de quatre thèmes: 1) Le métier d'agriculteur : points forts et points faibles du métier, évolution, outils de travail ;2) Le sol et la terre: évaluation de ces éléments naturels qui constituent le principal outil de travail ; 3) Le développement durable et l'agriculture durable: définition des concepts, médiatisation, appropriation par la société ; 4) Les politiques agricoles : évaluation des politiques et du système de subvention au niveau national, européen et mondial. Pour cette analyse, nous nous focaliserons essentiellement sur le troisième thème.

Concernant l'échantillon, les agriculteurs ont été sélectionnés en fonction de leur appartenance ou non à des groupements qui œuvrent en faveur de l'environnement. Trois formes d'agriculture ont été sélectionnées: l'agriculture conventionnelle, le GAB (Groupement d'Agriculture Biologique) et une forme d'agriculture durable, BASE (Bretagne-Agriculture-Sol-Environnement). Cette dernière, moins connue, dépend de l'Association pour la Promotion d'une Agriculture Durable $\left(A P A D^{1}\right)$. Elle fonde principalement son action sur la promotion de techniques de conservation des sols. Cette agriculture se veut productive et respectueuse de l'environnement tout en conservant l'utilisation de produits chimiques, à l'inverse de l'agriculture biologique. Les membres de ces deux réseaux ont été recrutés à partir des listes fournies par les administrateurs. Sur la base de ces listes, les agriculteurs ont été contactés aléatoirement. L'ensemble des personnes contactées a accepté l'entretien. Les agriculteurs conventionnels, quant à eux, ont été sélectionnés par l'intermédiaire de réseaux de connaissance. Toutes ces personnes ont été rencontrées à leur domicile. Au total, 21 agriculteurs ont participé à cette étude. La moyenne d'âge des personnes, majoritairement des hommes (19/21), est de 46 ans, s'échelonnant de 33 à 62 ans. Ce sont pour moitié des cultivateurs-éleveurs (11/21), les éleveurs étant en minorité (3/21). Dans l'ensemble, ces agriculteurs travaillent sur des petites exploitations ( 15 personnes possèdent moins de 100 ha). En termes de groupements, 8 personnes font partie du GAB, 7 personnes de l'association BASE et 6 personnes sont en conventionnel. Notre échantillon n'est pas représentatif des exploitations agricoles de Bretagne. Les résultats sont discutés par rapport à l'échantillon interrogé.

\subsection{Le choix des articles de presse}

10 En rapport à notre terrain d'étude, la Bretagne, nous avons choisi de porter notre analyse sur deux journaux régionaux : le quotidien d'actualité générale, Ouest France (OF), et l'hebdomadaire spécialisé dans l'agriculture, Paysan Breton (PB). Pour Ouest France, les articles contenant dans leur titre les expressions "développement durable», « agriculture durable », « durable » et/ou « durabilité » ont été récupérés. La sélection des textes basée sur les titres permet de s'assurer que le développement durable ou l'agriculture durable est l'objet central de l'article en question et qu'il apparait comme tel 
aux yeux du lecteur. D'autre part, nous avons limité notre sélection aux papiers de plus de 400 mots afin d'éliminer les communiqués annonçant des événements (foires, manifestations, expositions, etc.), moins porteurs de sens, au vu de nos objectifs. Le choix des articles pour ce quotidien s'est effectué à partir de la base de données électronique Factiva $^{\oplus}$, base de données spécialisée dans le recensement d'articles de presse d'information générale et spécialisée. Nous avons récolté l'ensemble des articles disponibles, à savoir 84 articles depuis novembre 2002 jusqu'à la période d'entretien, juin 2008. Concernant Paysan Breton, nous avons récupéré l'ensemble des articles faisant référence aux mêmes thèmes que ceux du Ouest France, disponibles dans les archives du site Internet de l'hebdomadaire. Au total, 13 articles sont apparus entre 2001 et 2008, contenant tous dans leur titre l'une des expressions inductrices même si, à la base, la sélection ne permettait pas de préciser ce choix.

\subsection{Analyse de contenu des documents}

11 Les corpus, articles mis en forme et entretiens intégralement retranscrits, ont été soumis à des analyses de données textuelles assistées par ordinateur à l'aide du logiciel Alceste (Reinert, 1990). Ce logiciel utilise la méthode de la Classification Descendante Hiérarchique $(\mathrm{CDH})$ en procédant par fractionnements successifs du texte, permettant de dégager des classes de mots représentatifs du discours. Le khi-deux calculé pour chaque mot indique son lien statistique, plus ou moins fort, avec la classe. L'analyse peut également faire apparaître des distinctions au sein des classes obtenues selon certaines variables discriminantes retenues (en l'occurrence le type d'agriculture pratiquée dans le cas des entretiens) qui n'interviennent pas directement dans le processus d'analyse. Le but de ces analyses est d'appréhender les discours dans leur intégralité et d'en dégager les grandes thématiques.

Dans le cadre de ces analyses, nous avons fait le choix de réaliser trois analyses distinctes. Nous avons d'une part distingué le corpus d'articles de presse de celui des entretiens. L'analyse du discours des agriculteurs a été complétée par une analyse manuelle, permettant une approche plus qualitative du contenu. D'autre part, concernant les articles de presse, nous avons également fait le choix de procéder à deux analyses distinctes en raison de contenus différents : les textes publiés au sein du Paysan Breton sont en moyenne un peu plus longs (PB : 587 mots en moyenne; OF : 470 mots en moyenne) et beaucoup moins nombreux (PB : 13 articles; OF : 84 articles). Des analyses disjointes s'imposaient afin de faire apparaître les spécificités propres à chaque corpus.

\section{Résultats}

\subsection{Le développement et l'agriculture durables dans la presse}

Les résultats de l'analyse Alceste, pour la presse, sont présentés ci-dessous. Le tableau 1 recense les mots les plus récurrents au sein de l'ensemble des deux corpus. À l'évidence, les mots-clés utilisés pour la sélection ("développement ", "agriculture », « durable ») sont les plus fréquents. Les deux piliers «environnemental » et «économique » sont présents au sein des corpus et le concept est envisagé dans l'action (" action », « faire »). Malgré ces points communs, nous pouvons noter quelques distinctions qui reflètent la vocation de chaque journal. 
Tableau 1. Effectifs des 20 termes les plus fréquents par ordre décroissant

\section{Ouest France}

\begin{tabular}{|lll|}
\hline \multicolumn{1}{|c}{ Formes réduites $^{(1)}$} & Effectifs $^{(2)}$ & $\%^{(3)}$ \\
\hline Durable & $405^{(4)}$ & 17 \\
développement & 386 & 16 \\
agriculture & 132 & 6 \\
faire & 120 & 5 \\
environnement & 114 & 5 \\
économie & 107 & 4 \\
action & 105 & 4 \\
énergie & 97 & 4 \\
entreprise & 95 & 4 \\
conseil & 95 & 4 \\
an & 84 & 4 \\
aller & 79 & 3 \\
général & 78 & 3 \\
fait & 77 & 3 \\
ville & 77 & 3 \\
premier & 71 & 3 \\
eau & 67 & 3 \\
politique & 66 & 3 \\
social & 65 & 3 \\
mettre & 63 & 3 \\
\hline
\end{tabular}

Paysan Breton

\begin{tabular}{|lcc|}
\hline Formes réduites & Effectifs & $\%$ \\
\hline durable & 69 & 14 \\
agriculture & 68 & 14 \\
développement & 41 & 8 \\
productif & 35 & 7 \\
produit & 25 & 5 \\
exploiter & 23 & 5 \\
environnement & 21 & 4 \\
économie & 21 & 4 \\
aliment & 20 & 4 \\
année & 19 & 4 \\
faire & 18 & 4 \\
élever & 18 & 4 \\
également & 16 & 3 \\
action & 16 & 3 \\
lait & 15 & 3 \\
qualité & 15 & 3 \\
système & 15 & 3 \\
objectif & 15 & 3 \\
filière & 14 & 3 \\
ministre & 14 & 3 \\
\hline
\end{tabular}

Notes: (1) Les formes réduites correspondent aux racines des termes. Par exemple, sous le terme " agriculture » se retrouvent également les termes « agriculteur(trice)(s). (2) L'effectif correspond à la fréquence d'apparition du terme au sein du corpus classé. (3) Le pourcentage est calculé en rapport à la fréquence totale des 20 mots présentés. (4) Les lignes grisées contiennent les mots communs aux deux corpus.

Ouest France, presse généraliste à grande diffusion, traite en premier lieu du « développement » durable. Le concept est abordé de manière globale et les trois piliers sont explicitement ("environnement», «économie », "social») ou implicitement (« eau », « énergie», «entreprise », etc.) présents. L'aspect politique est également évoqué ("politique», «Conseil général»). Les verbes d'actions sont généralistes ( «mettre », « faire », « aller »). À l'inverse et sans surprise, Paysan Breton, hebdomadaire spécialisé, renvoie en premier lieu à l'«agriculture» durable. Les verbes s'inscrivent davantage dans l'action liée à l'agriculture (« exploiter», "élever»). La dimension économique domine ("productif », " produit », « économie », « filière »), au détriment des autres dimensions. La dimension environnementale apparaît uniquement à travers le terme "environnement» et la dimension sociale est inexistante. Une analyse plus détaillée du contenu de chaque journal est présentée ci-dessous.

\section{La presse régionale généraliste (Ouest France)}

Sur la base de $74 \%$ de corpus classé, la classification descendante hiérarchique (CDH) met en exergue quatre classes qui permettent de dégager les principales thématiques (figure 1). Pour des raisons de présentation, pour cette figure et les suivantes, seuls les 24 premiers mots ayant les $\mathrm{khi}^{2}$ les plus élevés peuvent être affichés. La classe 4 , qui regroupe $30,65 \%$ du contenu analysé, traite du rôle de l'agriculture ; la classe 3 porte sur la politique territoriale $(29,09 \%)$; la classe 1 fait référence à l'écologie urbaine $(24,16 \%)$; 
et la classe 2 renvoie à la définition de la durabilité et plus précisément au développement durable $(16,10 \%)$.

La classe 4, qui porte sur le thème de l'agriculture, est la plus représentée au sein du corpus. L'agriculture, définie comme durable, réfère à une agriculture rurale (" ferme ", «paysan », « rural »), familiale (« famille » $\left[\mathrm{khi}^{2}=11\right]$, « couple » $\left[\mathrm{khi}^{2}=9\right]$, personnalisation par l'emploi de prénoms, par exemple « Marc » $\left.\left[\mathrm{khi}^{2}=9\right]^{2}\right)$. C'est une agriculture ouverte sur le monde extérieur ("valoriser») et associée à des pratiques écologiques («bois», réduction des "engrais » et des "pesticides" $\left[\mathrm{khi}^{2}=12\right]$, "panneau solaire », «bio » $\left.\left[\mathrm{khi}^{2}=12\right]\right)$. Les initiatives, liées au développement durable, sont également mises en avant (« Civam $\left.^{3} », « \mathrm{CAD}^{4} »\right)$.

Figure 1. Aperçu des résultats de la $\mathrm{CDH}$ (Alceste) pour les articles du Ouest France et interprétation thématique des classes

\begin{tabular}{|c|c|c|c|c|c|c|c|}
\hline \multirow{3}{*}{\multicolumn{2}{|c|}{ Classe 1}} & \multicolumn{3}{|c|}{ Unités de texte classées } & & \multirow{2}{*}{\multicolumn{2}{|c|}{$\prod_{\substack{\text { Classe } 4 \\
30,65 \%}}$}} \\
\hline & & \multicolumn{2}{|c|}{$\begin{array}{l}\text { Classe } 2 \\
16,10 \% \\
\end{array}$} & \multicolumn{2}{|c|}{$\begin{array}{l}\text { Classe } 3 \\
29,09 \% \\
\end{array}$} & & \\
\hline & & Présence & khi2 & Présence & khi2 & Présence & khi2 \\
\hline ville & 53 & \begin{tabular}{|l} 
futur \\
\end{tabular} & 53 & conseil & 154 & agriculture & 137 \\
\hline transport & 50 & entreprise & 45 & général & 119 & exploiter & 96 \\
\hline construction & 39 & planète & 43 & Maille & 53 & ferme & 79 \\
\hline vélo & 29 & génération & 36 & Agenda 21 & 40 & productif & 61 \\
\hline eau & 27 & ambigu & 32 & programme & 33 & laitier & 51 \\
\hline tri & 25 & salarié & 32 & Lorient & 29 & Civam & 40 \\
\hline déchet & 25 & société & 32 & Finistère & 25 & agricole & 34 \\
\hline prise & 23 & capacité & 32 & lancer & 23 & paysan & 31 \\
\hline national & 23 & mondial & 31 & président & 22 & hectare & 25 \\
\hline CUA & 22 & développement & 30 & élu & 21 & herbe & 24 \\
\hline énergie & 22 & ressource & 28 & projet & 21 & nural & 24 \\
\hline agglomération & 21 & humain & 26 & opposition & 21 & chiffre & 23 \\
\hline conscience & 20 & réponse & 26 & savoir & 20 & euro & 22 \\
\hline santé & 19 & définition & 25 & développement & 20 & produit & 21 \\
\hline inciter & 19 & humanité & 21 & charte & 19 & bois & 20 \\
\hline déplacement & 18 & scientifique & 21 & siècle & 18 & surface & 17 \\
\hline Brest & 17 & besoin & 19 & cap & 17 & milieu & 16 \\
\hline urbain & 17 & sens & 18 & Orient & 17 & engrais & 16 \\
\hline réduire & 17 & responsable & 18 & session & 17 & solaire & 16 \\
\hline quartier & 17 & Ory & 16 & régional & 17 & prairie & 16 \\
\hline pollution & 17 & Seine & 16 & CJD & 15 & litre & 15 \\
\hline doux & 16 & tirer & 16 & décider & 15 & panneau & 15 \\
\hline sélectif & 16 & sauce & 16 & François & 15 & valoriser & 15 \\
\hline littoral & 16 & boite & 16 & transversal & 15 & $\mathrm{CAD}$ & 14 \\
\hline
\end{tabular}

Note : Les pourcentages correspondent à la proportion de chaque classe thématique dans l'ensemble du corpus classé $(74 \%)$.

La classe 3, classe suivante la mieux représentée, aborde le « durable » sous l'angle de la politique territoriale. Les citations de Pierre «Maille», «Président» du «Conseil Général » du «Finistère » depuis 1998, sont redondantes. Sont mises en avant les initiatives de développement durable réalisées par les «élus» départementaux, notamment l'«Agenda $21 »^{5}$. Le développement durable renvoie ainsi aux "programmes » d'action mis en œuvre localement par les collectivités territoriales. Il constitue un enjeu politique basé sur la mise en valeur des actions et la sensibilisation des citoyens.

Le développement durable et l'agriculture durable renvoient également à l'écologie urbaine (classe 1), opposée à l'« écologie rurale » (classe 4). Cette classe fait référence à l'aménagement des villes et des transports («ville», «transport», «construction», 
« vélo », « agglomération », « déplacement », « quartier », «CUA », etc.). Elle traite également des pratiques écologiques (économie d'« eau ", "tri des déchets ", économie d'« énergie » et « énergie » renouvelable, « geste » éco-citoyen [ $\left.\mathrm{khi}^{2}=13\right]$, etc.) en lien avec la gestion des problèmes environnementaux (" eau », gestion du "littoral, "rivière » [khi $\left.{ }^{2}=12\right]$, etc.). Les initiatives de développement durable des grandes villes de l'Ouest, associées à une gestion écologique, sont mises en lumière. Le caractère environnemental prédomine par rapport aux autres piliers.

19 La classe 2, quant à elle, interroge la définition du durable et plus particulièrement du développement durable. Le concept apparaît comme un objet nouveau, «ambigu », à la "mode » $\left[\mathrm{khi}^{2}=14\right]$ et qui ne fait pas toujours «sens ». La référence à la définition du rapport Brundtland est fréquente ("futur», "génération», "capacité", "développement", "besoin»). Le concept est conçu comme global ("planète", "société », «mondial», «humanité») et met en exergue la «responsabilité » de tous dans la préservation des « ressources ». Le développement durable est par conséquent un concept interrogé du point de vue de son sens, de ce à quoi il renvoie en termes d'« éthique » $\left[\mathrm{khi}^{2}=10\right]$ et d'équité (« équitable » $\left[\mathrm{khi}^{2}=6\right]$ ), de l'ambiguïté qu'il sous-tend, et de ses éventuelles alternatives. Pour exemple, cet extrait tiré d'un article paru dans Ouest France le 30 mai 2006 : "C'est l'expression insupportable par excellence, vide de sens pour nombre d'entre nous, incapables de dire à quoi concrètement elle se rapporte. Formule à la mode, accusatoire et publicitaire à la fois, le développement durable n'est pourtant déjà plus le simple cache-misère d'une société de consommation en délicatesse avec sa bonne conscience ».

\section{La presse régionale spécialisée (Paysan Breton)}

Sur la base de $61 \%$ de textes classés, l'analyse Alceste permet de dégager quatre principales thématiques (figure 2). Par ordre d'importance, la classe 1 regroupe $44,27 \% \mathrm{du}$ contenu analysé ; elle traite de la production agricole et principalement de l'élevage. La classe 3 porte sur la durabilité $(20,61 \%)$. La classe 4 fait référence à la valorisation du produit et donc à la question économique (19,85\%). Et la classe 2 évoque la qualité du produit, et en l'occurrence le bien-être animal dont elle dépend (15,27\%). 
Figure 2. Aperçu des résultats de la $\mathrm{CDH}$ (Alceste) pour les articles du Paysan Breton et interprétation thématique des classes

\begin{tabular}{|c|c|c|c|c|c|c|c|}
\hline & \multicolumn{4}{|c|}{ Unités de texte classées } & & & \\
\hline \multicolumn{2}{|c|}{$\begin{array}{l}\text { Classe } 1 \\
44,27 \% \\
\end{array}$} & \multicolumn{2}{|c|}{$\begin{array}{l}\text { Classe } 2 \\
15,27 \%\end{array}$} & \multicolumn{2}{|c|}{$\begin{array}{l}\text { Classe } 3 \\
20,61 \% \\
\end{array}$} & \multicolumn{2}{|c|}{$\begin{array}{l}\text { Classe } 4 \\
19,85 \%\end{array}$} \\
\hline Présence & khi2 & Présence & khi2 & Présence & khi2 & Présence & khi2 \\
\hline élever & 14 & animal & 41 & développement & 39 & prix & 30 \\
\hline élevage & 12 & prioritaire & 23 & agriculture & 37 & frais & 25 \\
\hline herbe & 9 & objectif & 20 & durable & 35 & part & 21 \\
\hline social & 8 & santé & 17 & homme & 24 & chiffre & 21 \\
\hline travail & 8 & nouveau & 13 & eau & 23 & collecter & 17 \\
\hline simplifier & 7 & recherche & 13 & environnement & 18 & volaille & 17 \\
\hline produire. & 7 & aliment & 11 & idée & 11 & affaire & 16 \\
\hline exploiter & 7 & coût & 11 & monde & 11 & revient (prix) & 16 \\
\hline souhait & 7 & projet & 11 & ministre & 10 & euro & 16 \\
\hline laitier & 5 & agricole & 8 & plan & 7 & continuer & 12 \\
\hline prévoir & 5 & notamment & 6 & faire & 5 & baisse & 12 \\
\hline administratif & 5 & adaptatif & 6 & apparaittre & 5 & céréale & 12 \\
\hline procédure & 5 & humain & 6 & Bretagne & 5 & année & 11 \\
\hline structure & 5 & rester & 6 & progressif & 4 & résultat & 10 \\
\hline mise & 4 & répondre & 6 & mettre & 4 & bovin & 8 \\
\hline moyen & 4 & innover & 6 & présent & 4 & lait & 8 \\
\hline pratique & 4 & INRA & 6 & problème & 4 & A & 6 \\
\hline azote & 4 & scientifique & 6 & salon & 4 & pays & 5 \\
\hline financement & 4 & action & 5 & équilibre & 4 & voie & 4 \\
\hline épandage & 4 & vétérinaire & 4 & fait & 3 & société & 4 \\
\hline gestion & 4 & Guillou & 4 & terre & 3 & métier & 4 \\
\hline autonomie & 4 & hausse & 4 & territoire & 2 & stratégie & 4 \\
\hline service & 4 & norme & 4 & ZES & 2 & commercial & 4 \\
\hline forme & 4 & nombre & 4 & breton & 2 & coopératif & 4 \\
\hline
\end{tabular}

21 La classe 1, la mieux représentée dans le corpus, traite des procédés de production et principalement d'élevage, première production agricole de Bretagne (Agreste, 2009) ${ }^{7}$. En rapport à la production, la durabilité est évoquée dans toutes ses dimensions. La dimension "sociale " transparaît à travers la gestion des ressources humaines mais également la qualité de vie, parfois difficile selon le type d'exploitation (par exemple, obligation des traites journalières en production laitière). La dimension environnementale est évoquée, plutôt sous forme de désagrément, en référence aux contraintes « administratives » engendrées, à la complexité et au coût des " procédures ». Sont également citées les pratiques en matière d'épandage ("pratique», "azote", " épandage »). Enfin la dimension économique renvoie au « financement » des structures, à l'«autonomie » financière permettant une meilleure efficacité et donc durabilité de l'exploitation. Sont donc évoqués ici les éléments qui peuvent favoriser ou entraver la pérennisation de l'exploitation.

La classe 3 concerne les concepts de développement durable et d'agriculture durable. La dimension « environnementale » apparaît explicitement et implicitement en rapport à un problème central et une priorité du développement durable: l'« eau». Le problème (pénurie, qualité, etc.) est examiné tant au niveau mondial («monde») que local («Bretagne »). Mais bien que la référence à la globalisation du concept «durable » soit présente comme dans la presse généraliste, l'accent est mis ici sur l'aspect territorial ("Bretagne ", «breton », « territoire»). Les initiatives et les "plans » d'action sont mis en avant, qu'ils soient politiques («ministre», «ZES ») ou associatifs («salon» de la «terre »). Le développement durable y est présenté comme une recherche d'équilibre dans la relation entre l'homme et son environnement, au sens écologique du terme, dimension qui prédomine largement au sein des articles. 


\section{différemment les concepts de durabilité, conformément à leur vocation et au regard des} préoccupations qui leur sont propres. La presse généraliste (OF) diffuse le concept de développement durable dans le sens commun en s'appuyant sur des documents scientifiques ou d'experts (rapport Brundtland, 1987). Elle met l'accent sur la dimension écologique du développement durable (prévalence du pilier environnemental) et sur des lieux communs, opposant ainsi l'écologie rurale à l'écologie urbaine. La dimension politique (locale) est également très présente à travers les initiatives des villes, des départements et de la région (Bretagne). Elle est le reflet des actions concrètes. La presse spécialisée (PB) s'approprie le concept en traduisant le développement durable en termes d'agriculture durable. Conformément au sens commun, la dimension environnementale est manifeste mais la dimension économique semble prévaloir avant tout : la durabilité de l'agriculture est rendue possible par l'autonomie financière des exploitations, le rapport qualité/prix de la production, la valorisation économique de la production, etc. Néanmoins, comme nous l'avons précédemment soulevé, la dimension sociale est très peu abordée. Nous allons maintenant nous attarder sur le contenu des discours des agriculteurs.

\subsection{Le développement et l'agriculture durables dans le discours des agriculteurs}

Parmi les $71 \%$ de textes classés, l'analyse Alceste permet de dégager trois classes thématiques dans le discours des agriculteurs (figure 3). Par ordre d'importance, la classe 2 fait référence à l'agriculture durable et au développement durable interrogés dans leur sens et sous l'angle du changement; elle représente $75,27 \%$ du corpus classé. La classe 1 porte sur les pratiques et les modes de consommation (15,38\%). Et la classe 3 renvoie à la définition de la durabilité à travers ses trois piliers: l'environnement, le social et l'économique $(9,64 \%)$. 
Figure 3. Aperçu des résultats de la $\mathrm{CDH}$ (Alceste) pour les entretiens et interprétation thématique des classes

\begin{tabular}{|c|c|c|c|c|c|}
\hline & & Unités de text & assées & & \\
\hline $\begin{array}{l}\text { Classe } \\
15,38\end{array}$ & & $\begin{array}{l}\text { Classe } \\
75,27\end{array}$ & & $\begin{array}{c}\text { Classe } 3 \\
9,64 \%\end{array}$ & \\
\hline Présence & khi2 & \begin{tabular}{|l|} 
Présence \\
\end{tabular} & khi2 & Présence & khi2 \\
\hline voiture & 55 & \begin{tabular}{|l} 
durable \\
\end{tabular} & 10 & social & 141 \\
\hline supermarché & 34 & parler & 6 & aspect & 89 \\
\hline conscience & 34 & utiliser & 6 & environnemental & 69 \\
\hline compte & 33 & changer & 5 & critère & 61 \\
\hline producteur & 29 & agriculture & 5 & économique & 60 \\
\hline collectif & 28 & société & 5 & cahier & 49 \\
\hline client & 28 & pratique & 4 & charge & 49 \\
\hline boulot & 28 & bio & 4 & action & 42 \\
\hline local & 28 & différent & 4 & ferme & 39 \\
\hline prise & 28 & développement & 4 & lien & 30 \\
\hline acheter & 26 & respect & 4 & proximité & 30 \\
\hline émission & 22 & an & 4 & hectare & 29 \\
\hline télé & 17 & grand & 3 & SMIC & 21 \\
\hline jour & 17 & revenir & 3 & poulet & 21 \\
\hline essence & 17 & vivre & 3 & échange & 15 \\
\hline rendre & 16 & nourrir & 3 & investir & 12 \\
\hline énergie & 16 & poser & 3 & fonctionner & 12 \\
\hline cher & 14 & paysan & 3 & écologique & 12 \\
\hline euro & 12 & savoir & 3 & énergétique & 12 \\
\hline bonne & 11 & terme & 3 & nombre & 8 \\
\hline nourriture & 11 & mot & 3 & tracteur & 8 \\
\hline prix & 11 & sol & 3 & difficile & 8 \\
\hline fin & 10 & système & 3 & efficace & 8 \\
\hline consommation & 9 & wraiment & 3 & permettre & 8 \\
\hline
\end{tabular}

La classe 2, la mieux représentée, reflète un fort questionnement sur le sens même du «durable» ("parler», " poser», «mot», « question» [ $\left.\mathrm{khi}^{2}=2\right]$ ). Le concept apparait comme une notion indéfinissable, un «terme ", un « mot », dénué de sens, galvaudé, à la mode, utilisé à toutes les sauces; ces expressions sont redondantes dans le discours des agriculteurs. Pour être durable, le « changement » de «pratiques » semble nécessaire, au niveau de la « société » dans son ensemble et pas seulement au sein du monde « paysan ». Néanmoins, l'« agriculture » a particulièrement un rôle à jouer (préservation de l'outil de travail - le «sol », « nourrir » les hommes, etc.). Conceptuellement, l'agriculture «bio » représente l'agriculture durable par excellence, mais en pratique, ses quantités de production restent limitées pour nourrir durablement la planète. Cette classe est caractéristique des agriculteurs de BASE $\left[\mathrm{khi}^{2}=15\right]$ et des agriculteurs conventionnels $\left[\mathrm{khi}^{2}=14\right]$. Nombreux agriculteurs expriment un certain agacement lié à l'utilisation de ce terme par le sens commun (et notamment dans les médias). «Il y a du durable à toutes les sauces " (Conventionnel, 17) ${ }^{11}$, "On en parle beaucoup mais pas forcément à bon escient " (BASE, 1). La notion de " croissance » $\left[\mathrm{khi}^{2}=2\right]$ est également associée à cette classe. Pour les agriculteurs de BASE notamment, les termes « développement » et « croissance » vont de pair. Pour eux, la durabilité correspond avant tout à une pérennisation de l'exploitation, de l'outil de travail ( « sol »). La dimension économique est très présente, « celui qui est durable, c'est celui qui passera la crise " (BASE, 2). L'agriculture ne peut être durable qu'à partir du moment où elle est productive, notamment parce qu'elle doit permettre de « nourrir » la planète, «Quand on sait qu'il faudra nourrir 9 milliards d'habitants en 2150 » (BASE, 3), ce que justement le «bio » ne permet pas «c'est prouvé (...) qu'on pourra pas nourrir 9 milliards d'habitants qu'avec du bio » (BASE, 1). 

été défini par les organisations internationales (rapport Brundtland, 1987 ; Conférence de Rio, 1992) : un développement basé sur trois piliers : "social », « environnemental » et "économique ». Le «social» porte sur la nécessité de créer ou de maintenir du lien social, des échanges locaux (" proximité») et des échanges internationaux raisonnés en évitant l'importation de produits fabriqués en France ou l'utilisation de céréales étrangères (par exemple, le soja d'Afrique pour nourrir les «poulets » de France). Ce discours, en lien avec la classe, précédente, est très présent chez les agriculteurs biologiques [ $\left.\mathrm{khi}^{2}=38\right]$. « Penser commerce de proximité, penser échanges avec les locaux. (...) C'est essayer de fonctionner comme ça, par du commerce local, du lien social aussi local. » (GAB, 19). La dimension économique est en lien avec la dimension sociale, c'est l'économie locale, c'est tirer un revenu de sa production afin d'assurer la pérennité de l'exploitation.

\section{Discussion}

30 Cette étude traite de l'appropriation du concept de développement durable par les agriculteurs, acteurs en prise avec ce sujet. Dans leur discours, le développement durable est questionné du point de vue de son sens. Les trois piliers sont connus, mais les agriculteurs s'interrogent sur la cohérence entre la conception idéologique du développement durable (dominée par la dimension environnementale) et les modes de consommation de la société occidentale actuelle. Ce discours peut être mis en parallèle avec le contenu affiché au sein des articles du Ouest France. Cette presse généraliste informe sur la définition du développement durable, met en avant l'importance de la dimension environnementale et met en exergue des exemples de pratiques écologiques. En conséquence, les agriculteurs discutent de ces thèmes et soulèvent des questions liées à la sensibilisation, à la prise de conscience et aux pratiques éco-citoyennes. Ces réflexions se retrouvent également au cœur des articles publiés dans le Paysan Breton, presse spécialisée. Les agriculteurs pointent ainsi les difficultés d'appropriation de ce concept. Par conséquent, son imprécision renvoie à un terme galvaudé dont on ne retient que les expressions de croissance, de changement, de projection dans le futur, etc..

31 En termes d'agriculture durable, les positions semblent plus claires. Certains agriculteurs définissent avant tout l'agriculture durable comme une agriculture locale dans sa production et de proximité avec le client (c'est notamment le cas des agriculteurs 
biologiques), d'autres caractérisent l'agriculture durable comme une agriculture productive (principalement les agriculteurs de BASE et les agriculteurs conventionnels).

Pour les premiers, majoritairement des agriculteurs biologiques, une agriculture durable doit favoriser la préservation des ressources naturelles dans leur ensemble. Cette préservation passe par la non-utilisation de produits chimiques. C'est une agriculture qui se définit par une économie avant tout locale et qui se veut socialement proche du consommateur. En rapport à la presse, ce premier groupe se rapproche de la conception de l'agriculture (écologie rurale) telle que définie dans la presse généraliste. Autrement dit, pour ces agriculteurs mais également pour le sens commun, l'agriculture durable est le modèle de l'agriculture biologique par excellence (respectueuse de l'environnement, associée à des pratiques écologiques, etc.).

Le second groupe insiste davantage sur l'importance du terme "développement ». Une agriculture durable doit être productive, d'une part pour permettre de nourrir l'ensemble de la population mondiale, et d'autre part pour simplement subsister. Dans ce cas, la dimension de développement économique prévaut. En rapport à l'analyse de presse, ce discours se retrouve au sein de la presse spécialisée qui, comme nous avons l'avons constaté, met en avant l'importance de l'autonomie financière, du rapport entre qualité et coût de production, de la valorisation des produits, etc. Pour être durable, l'agriculture doit être économiquement viable. Les autres dimensions du développement durable ne sont pas exclues, notamment la dimension environnementale : cette pérennisation va de pair avec un respect de la «terre », du «sol », conçu comme l'outil de travail. Il s'agit donc de participer à la croissance tout en respectant l'environnement, à l'inverse des agriculteurs biologiques. Nous pouvons ici souligner que Paysan Breton est le reflet de l'idéologie agricole majoritaire, éloignée des conceptions idéologiques des agriculteurs biologiques. Ces derniers sont souvent marginalisés par les membres des autres groupes agricoles et renvoyés à une image péjorative d' "écolos ", " ils sont bien gentils mais des fois je me demande si on fait le même métier. (...) Pour eux, quelques cochons et c'est bon " (Conventionnel, 18). Ils sont jugés comme des "idéalistes", contrairement aux agriculteurs attachés à des valeurs économiques qui se considèrent comme "réalistes", autrement dit qui estiment être dans la « réalité du sens commun », de la majorité. Une réalité où la dimension économique fait force et rime avec « croissance ", une réalité qui s'oppose à la décroissance, portée par une minorité.

La représentation sociale de l'agriculture durable est par conséquent conditionnée par la conception que les agriculteurs ont de leur métier (Michel-Guillou, 2006 ; Michel-Guillou, Moser, 2006 ; Weiss, Moser, Germann, 2006). Les membres de l'agriculture biologique s'attachent à des aspects tels que la sensibilisation environnementale, l'importance des comportements écologiques, la défense du milieu naturel et des paysages, etc. Ces dimensions sont partie prenantes du métier et sont au fondement même de leurs pratiques (Michel-Guillou, 2006). Ainsi, les aspects environnementaux et sociaux (proximité, lien social, etc.) du développement durable sont largement assimilés par ces derniers. Pour les agriculteurs de BASE, comme pour les agriculteurs conventionnels, le versant économique prime. La préservation écologique est également présente (protection des sols) mais surtout en rapport à la préservation d'un outil de travail et non comme une fin en soi. Les membres de BASE, visiblement insérés dans un groupe à caractère pro-environnemental, revendiquent le fait d'être inscrits dans une démarche respectueuse de l'environnement qui leur procure un confort de vie que, selon eux, ils ne pourraient acquérir en s'engageant dans une démarche d'agriculture biologique. Ce 
comportement peut se comprendre par la volonté des ces agriculteurs de se créer ou de maintenir une identité sociale positive (Tajfel, Turner, 1985) en se comparant et en se démarquant tout à la fois d'un groupe socialement reconnu, les agriculteurs biologiques, « beaucoup de gens sont persuadés que je fais du bio... » (BASE, 1).

Les agriculteurs conventionnels, quant à eux, ne sont pas explicitement rattachés à un groupe. Il semble résider parmi eux un foisonnement de modes de pensée, qui peuvent tout autant se rattacher au mode de pensée de l'agriculture biologique que celui de l'agriculture de conservation des sols.

\section{Conclusion}

Cette étude met en avant qu'il existe diverses représentations sociales du développement durable, différentes assimilations du concept, selon les pratiques mises en œuvre. Ces représentations sont elles-mêmes dépendantes d'idéologies et de conceptions différentes du métier qui guident les individus vers des actions privilégiées. En conclusion, il existe autant de définitions du développement durable qu'il existe de groupes agricoles, chacun s'appropriant l'objet de telle façon à le rendre conforme à ses propres modes de pensée.

\section{BIBLIOGRAPHIE}

Bonnes M. \& Bonaiuto M., 2002, "Environmental psychology: from spatial physical environment to sustainable development", in R.B. Bechtel \& A. Churchman (Eds.), Handbook of Environmental Psychology (pp. 28-54). New-York: Wiley and Sons.

Charvet J-P., 2007, « Agriculture et développement durable », in Y. Veyret (Ed.), Le développement durable (pp. 117-140). Paris : Sedes.

Commission mondiale sur l'environnement et le développement, 2005, Notre avenir à tous, Saint-

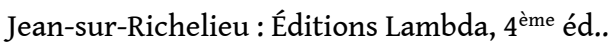

Doise W., 1985, « Les représentations sociales : définition d'un concept », Connexions, 45, 243-253.

Humbert A., 2008, « Développement durable et espace rural », in G. Wackermann (Ed.). Le développement durable (pp. 175-191). Paris : Ellipses.

Jodelet D., 1999, « Représentations sociales : un domaine en expansion », in D. Jodelet (Ed.), Les représentations sociales (6 ${ }^{\text {ème }}$ éd., pp. 47-78). Paris: PUF.

Jollivet M., 2001, Pour une science sociale à travers champs : Paysannerie, ruralité, capitalisme (France XXe siècle). Paris : Arguments.

Michel-guillou E., 2006, « Représentations et pratiques : l'exemple de l'engagement proenvironnemental en agriculture », Revue Européenne de Psychologie Appliquée, 56, 157-165.

Michel-Guillou E. \& Moser M., 2006, "Commitment of farmers to environmental protection: from social pressure to environmental conscience". Journal of Environmental Psychology, 26, 227-235. 
Michel-Guillou E. \& Weiss K., 2007, "Representations and behaviours of farmers with regard to sustainable development: A psycho-environmental approach", in B. A. Larson (Ed.), Sustainable development research advances (pp. 207-221). New York: Nova Science Publishers.

Moscovici S., 1976, La psychanalyse, son image et son public, Paris: PUF.

Moser G., 2009, Psychologie environnementale. Les relations homme-environnement. Bruxelles : De Boeck.

Oskamp S., 2000, "Psychological contributions to achieving an ecologically sustainable future for humanity", Journal of Social Issues, 56, 373-390.

Pol E., 2003, « De l'intervention à la gestion environnementale » in G. Moser G. \& K. Weiss, Espaces de vie. Aspects de la relation homme-environnement (pp. 305-329). Paris : Armand Colin.

Ratiu E., 2003, « L'évaluation de l'environnement », in G. Moser G. \& K. Weiss, Espaces de vie. Aspects de la relation homme-environnement (pp. 85-112). Paris : Armand Colin.

Reinert M., 1990, « Alceste, une méthode d'analyse des données textuelles. Application au texte "Aurélia" de Gérard de Nerval », Bulletin de Méthodologie Sociologique, 26, 25-54.

Schmuck P. \& Schultz W., 2002, "Sustainable development as a challenge for psychology", in P. Schmuck \& W. Schultz (Eds), The psychology of sustainable development (pp. 3-17), Norwell: Kluwer Academic Pub.

Tajfel H. \& Turner C., 1985, "The Social Identity Theory of Intergroup Behavior", in S. Worchel \& W.G. Austin (Eds), Psychology of Intergroup Relations (2 ème éd., pp. 7-24). Chicago: Nelson-Hall.

Weiss K. et Girandola F. (Eds), 2010, Psychologie et développement durable. Paris : In Press.

Weiss K., Moser G. \& Germann C., 2006, « Perception de l'environnement, conceptions du métier et pratiques culturales des agriculteurs face au développement durable », European Review of Applied Psychology, 56, 73-81.

\section{NOTES}

1. http://www.apad.asso.fr/sommaire.html

2. Chaque mot, présent au sein de la classe mais n'apparaissant pas sur la figure, est donné avec son $\mathrm{khi}^{2}$.

3. Centres d'Initiatives pour Valoriser l'Agriculture et le Milieu rural. Ces groupes s'inscrivent dans une action de développement durable. http://www.civam.org/

4. Contrat d'Agriculture Durable. Il a pour objectif de développer la multifonctionnalité de l'agriculture. http://agriculture.gouv.fr/contrats-d-agriculture-durable-cad

5. Les Agendas 21 sont des programmes d'actions de développement durable au niveau local. Ils sont apparus suite à la Conférence de Rio (1992) qui définissait les priorités (3 piliers) en matière de développement durable. Les actions locales à engager en faveur du développement durable se traduisent sous forme d'Agenda 21 mis en place par des collectivités territoriales. Celui du Finistère a débuté en 2003 par une phase de concertation avec les citoyens, les politiques, les associations, etc.. Il a été adopté en 2006 pour une période de 3 ans.

6. Communauté Urbaine d'Alençon.

7. D'après une enquête de l'Agreste menée en 2008 et publiée en 2009. http:// draf.bretagne.agriculture.gouv.fr/TAB-2009-et-TAB-2010

8. Arrêté de ZES (Zone d'excédents structurels) concernant l'épandage d'azote organique.

9. Institut National de Recherche Agronomique. 
10. Référence à Marion Guillou, directrice générale de l'INRA.

11. Entre parenthèses : le groupe d'appartenance (BASE / GAB / Conventionnel) et le numéro de l'entretien.

\section{RÉSUMÉS}

Cette étude porte sur la question du sens des concepts de développement durable et d'agriculture durable à travers, d'une part, une analyse de contenu de leur diffusion dans la presse régionale (presse spécialisée en milieu agricole et presse généraliste). D’autre part, elle s'intéresse à l'appropriation de ces concepts par les agriculteurs. Pour ce faire, des entretiens semi-directifs ont été menés auprès de ces acteurs en prise avec ces questions de « durabilité ». L'objectif est de mettre en parallèle ces deux formes de contenu et de mettre en lumière les représentations sociales liées à ces objets en partant du postulat que ces dernières, en tant que forme de connaissance socialement élaborées, sont un guide pour l'action et que celles-ci se forment, se transmettent, et évoluent au cœur de la communication (Moscovici, 1976).

This study focuses on the meaning of the concepts of sustainable development and sustainable agriculture. First, this study deals with a content analysis of their circulation in the regional papers (specialized in agriculture and popular newspaper). Second, it is interested in the appropriation of theses concepts farmers. Semi-structured interviews were carried out with these stakeholders concerned with these issues. The aim of the study is to compare these two content analyses and to highlight the social representations of these concepts. We postulate that these representations are a form of knowledge which is socially constructed. They are a guide to action and that they take shape, they are transmitted and they evolve in the heart of communication (Moscovici, 1976).

\section{INDEX}

Mots-clés : représentations sociales, agriculture durable, agriculteurs

Keywords : social representations, sustainable agriculture, farmers

\section{AUTEURS}

\section{LISE BIHANNIC}

Lise Bihannic est chargée d'études, diplômée du Master Psychologie sociale des représentations : études qualitatives et quantitatives (Université de Brest). Ses principaux domaines d'études portent sur les représentations sociales des concepts liés à l'environnement et au développement durable. Université de Brest, Université Européenne de Bretagne (UEB), EA 1285 - Centre de Recherches en Psychologie, Cognition et Communication, Institut des Sciences de l'Homme et de la Société (ISHS), 20, rue Duquesne - CS 93837, 29238 Brest cedex 3. Tél. : 02.98.01.63.61; Fax : 02.98.01.68.00 


\section{ÉLISABETH MICHEL-GUILLOU}

Élisabeth Michel-Guillou est maître de conférences à l'Université de Brest. Ses activités de recherche sont axées sur une approche psychosociale et environnementale du rapport de l'individu à l'environnement social et naturel et portent des thématiques telles que le développement durable, l'eau ou les changements climatiques. Université de Brest, Université Européenne de Bretagne (UEB), EA 1285 - Centre de Recherches en Psychologie, Cognition et Communication, Institut des Sciences de l'Homme et de la Société (ISHS), 20, rue Duquesne - CS 93837, 29238 Brest cedex 3. Tél. : 02.98.01.63.61 ; Fax : 02.98.01.68.00 Elisabeth.Michel-

Guillou@univ-brest.fr 